SUBJECT AREAS:

NANOPARTICLES

MATERIALS SCIENCE

Received

4 November 2013

Accepted

27 March 2014

Published

11 April 2014

Correspondence and requests for materials should be addressed to H.U.L. (leeho@kbsi.re.

kr) orJ.L. (jouhahn@ kbsi.re.kr)

* These authors contributed equally to this work.

\title{
Photoluminescent carbon nanotags from harmful cyanobacteria for drug delivery and imaging in cancer cells
}

Hyun Uk Lee ${ }^{1 *}$, So Young Park ${ }^{*}$, Eun Sik Park ${ }^{2 *}$, Byoungchul Son', Soon Chang Lee ${ }^{3}$, Jae Won Lee ${ }^{4}$, Young-Chul Lee ${ }^{5}$, Kyoung Suk Kang ${ }^{6}$, Moon II Kim ${ }^{5}$, Hyun Gyu Park ${ }^{6}$, Saehae Choi ${ }^{7}$, Yun Suk Huh ${ }^{8}$, Seung-Yeul Lee', Kyung-Bok Lee', You-Kwan Oh ${ }^{10}$ \& Jouhahn Lee'

'Division of Materials Science, Korea Basic Science Institute (KBSI), Daejeon 305-333, Republic of Korea, ${ }^{2}$ Korea Standards Research Institute of Food and Drug, Busan 607-120, Republic of Korea, ${ }^{3}$ Department of Applied Chemistry and Biological Engineering, Chungnam National University, Daejeon 305-764, Republic of Korea, ${ }^{4}$ Department of Energy Engineering, Dankook University, Cheonan 330-714, Republic of Korea, ${ }^{5}$ Department of BioNano Technology, Gachon University, 1342 Seongnamdaero, Sujeong-gu, Seongnam-si, Gyeonggi-do 461-701, Republic of Korea, ${ }^{6}$ Department of Chemical and Biomolecular Engineering (BK 21 program), KAIST, 291 Daehakno, Yuseong-gu, Daejeon 305-701, Republic of Korea, ${ }^{7}$ College of Pharmacy, Chungbuk National University, Cheongju 361-763, Republic of Korea, ${ }^{8}$ Department of Biological Engineering, College of Engineering, Inha University, Incheon 402-751, Republic of Korea, ${ }^{9}$ Division of Life Science, Korea Basic Science Institute (KBSI), Daejeon 305-333, Republic of Korea, ${ }^{10}$ Biomass and Waste Energy Laboratory, Korea Institute of Energy Research (KIER), 152 Gajeong-ro, Yuseong-gu, Daejeon 305-343, Republic of Korea.

Using a simple method of mass production of green carbon nanotags (G-tags) from harmful cyanobacteria, we developed an advanced and efficient imaging platform for the purpose of anticancer therapy.

Approximately 100 grams of G-tags per 100 kilograms of harmful cyanobacteria were prepared using our eco-friendly approach. The G-tags possess high solubility, excellent photostability, and low cytotoxicity $(<1.5 \mathrm{mg} / \mathrm{mL}$ for $24 \mathrm{~h})$. Moreover, doxorubicin-conjugated G-tags (T-tags; $>0.1 \mathrm{mg} / \mathrm{mL}$ ) induced death in cancer cells (HepG2 and MCF-7) in-vitro at a higher rate than that of only G-tags while in-vivo mice experiment showed enhanced anticancer efficacy by T-tags at $0.01 \mathrm{mg} / \mathrm{mL}$, indicating that the loaded doxorubicin retains its pharmaceutical activity. The cancer cell uptake and intracellular location of the Gand T-tags were observed. The results indicate that these multifunctional T-tags can deliver doxorubicin to the targeted cancer cells and sense the delivery of doxorubicin by activating the fluorescence of G-tags.

\footnotetext{
( ancer is one of the leading causes of human death worldwide. As a result, the principle challenges faced in anticancer therapy using nanotechnology have been the design and development of nanomaterials with multiple functions ${ }^{1-4}$. Anticancer therapy using multifunctional nanomaterials can target a cancer or tumour, deliver therapeutic drugs, and monitor the tumour tissues ${ }^{5-9}$. In recent years, the use of various types of biological labelling probes such as gold nanoshells ${ }^{10,11}$, semiconductor quantum dots $\mathrm{s}^{4,12}$, iron oxide nanoparticles $^{13,14}$, carbon nanotubes ${ }^{15}$, and polymer nanocarriers ${ }^{9,16}$ has resulted in the development of sensitive and specific targeted cell imaging, sensing, and therapy for in vitro and in vivo applications. However, many of those probes possess properties that considerably decrease their therapeutic efficacy, including poor photostability and solubility in aqueous media, poor biodistribution, and a lack of target selectivity ${ }^{5,17-20}$.

Alternatively, carbon-based multifunctional water-soluble nanomaterials can be fabricated from bulk biological sources into fluorescent probes, thus reducing ecological concerns and economic problems ${ }^{21,22}$. Recently, our group reported green carbon dots (G-dots) from food waste-derived sources as novel fluorescent probes for cell imaging. Although G-dots do not possess any targeting ligands on their surfaces, they are biocompatible and stable in aqueous systems, permitting their use in biomedical applications ${ }^{23}$. Compared to previous metallic or inorganic nanoparticles ${ }^{1,6,24}$, carbon dot delivery systems can be used to minimise cytotoxicity and improve clinical outcomes and comprise the next generation of multifunctional nanomedicine ${ }^{22,23,25}$. Nevertheless, there are several critical issues involved in the design of carbon nanomaterials ${ }^{26,27}$, such as surface functionalization. Past attempts have involved attachment of gold nanoparticles to carbon nanotubes, resulting in the production of large clusters, which were unsuitable for biomedical applications ${ }^{28}$. While these various imaging and therapeutic nanoparticles represent an exciting improvement in the field of biosensing and cell imaging, it would be ideal to
} 

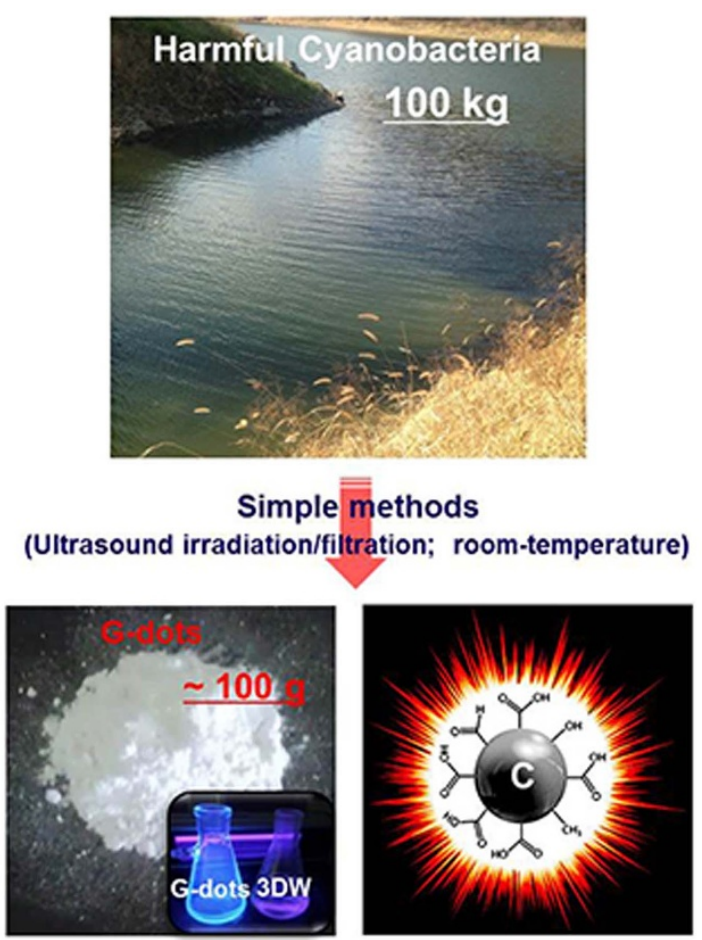

Figure 1 The available mass production of photoluminescent carbon nanotags from harmful cyanobacteria. Synthesis of G-tags from cyanobacteria biomass (approximately 100 grams of G-tags per 100 kilograms of cyanobacteria), using a simple and eco-friendly approach. The photograph of cyanobacteria was taken by Prof. Young-Chul Lee and the schematic graphic was done by Ms. So Young Park.

engineer 'advanced' versatile carbon dots that are capable of performing biological functions (e.g. sensing, imaging, and drug delivery) by a very simple manufacturing method ${ }^{29,30}$.

In this study, we describe the design of an advanced 'green solution' for the disposal of harmful green microalgae (Fig. 1). Highquality carbon materials were prepared from harmful cyanobacteria using our simple method (ultrasound irradiation/filtration processing) at room temperature, and they exhibited excellent photoluminescence properties, high photostability, size uniformity, and low cytotoxicity. In our simple method, not adding extra hydrophilic molecules resulted in high solubility and high drug loading capacity, since the functional groups themselves were hydrophilic. This provides an in vitro demonstration of therapeutic carbon dots (T-tags; multifunctional G-tags conjugated with doxorubicin [DOX]) suitable for cell imaging and anticancer drug delivery. We report a simple technology for T-tags that can be used to image and deliver anticancer drugs to cancer cells [HepG2 (hepatocellular liver carcinoma, human) and MCF-7 (breast cancer, human)] without photobleaching. These properties have opened new possibilities for synergistic therapeutic targeting as well as diagnostic imaging.

\section{Results}

Physicochemical, structural, and optical properties of G-tags. The morphological features of the G-tags (Fig. 2) were assessed using high-resolution transmission electron microscopy (HR-TEM) and atomic force microscopy (AFM). The images showed that the Gtag nanoparticles had a uniform spherical shape with a diameter of 1-11 nm, with most of the particles being 3-6 nm wide. The average G-tag particle size determined from the TEM and AFM images was approximately $5.7 \mathrm{~nm}$.

The X-ray diffraction (XRD) pattern of the G-tags (Fig. 3a) showed peaks at $2 \theta=24.7^{\circ}$ and $2 \theta=43.3^{\circ}$ that are assigned to the (002) and (101) diffraction patterns of graphitic carbon, respectively ${ }^{31,32}$. The former peak corresponds to the interlayer spacing of $\sim 3.77 \AA$, which is slightly longer than the spacing between the (002) planes in bulk graphite (3.44 $⿱$ ) ). The larger interlayer spacing (compared to bulk graphite) is ascribed to the existence of abundant functional groups. The XRD patterns and Raman spectrum of the G-tags showed that the resultant crude G-tags possess carbon structures, indicating an amorphous nature, as shown in Figs. 3a and $3 \mathrm{~b}$. The weak peak at $1580 \mathrm{~cm}^{-1}$ ( $\mathrm{G}$ band) corresponds to the $E_{2 g}$ mode of the graphite and is related to the vibration of $s p^{2}$-bonded carbon atoms in a two-dimensional (2D) hexagonal lattice and disordered carbon, which indicates an amorphous nature (Fig. 3b) ${ }^{31}$. The D band at around $1360 \mathrm{~cm}^{-1}$ is associated with the vibrations of carbon atoms with dangling bonds in the termination plane of disordered graphite or glassy carbon ${ }^{31}$. These results may be related to the carbon precursors and carbonisation processing, which are employed to simplify the methods (ultrasound irradiation and filtration processing), and the lack of a calcination process (at room temperature), and are in good agreement with our previous results. The FTIR spectrum was characterised to obtain structural insights regarding the G-tags, as shown in Fig. 3c. The Fourier transform infrared (FTIR) results for the G-tags showed the following: vibrations of the $\mathrm{OH}, \mathrm{C}-\mathrm{O}-\mathrm{C}, \mathrm{C}=\mathrm{O}$, and $\mathrm{C}-\mathrm{H}$ bonds, asymmetric and symmetric stretching vibrations of C-O-C (around 1300 and $1200 \mathrm{~cm}^{-1}$ ), and $\mathrm{C}=\mathrm{O}$ stretching vibrations were detected, indicating partial oxidation of the G-tag surface ${ }^{31-33}$. The broad absorption bands at $3400 \mathrm{~cm}^{-1}$ were assigned to - $\mathrm{OH}^{32,33}$. The G-tags exhibited very good solubility in water due to the abundant oxygen-containing groups or hydrophilic groups (e.g. - $\mathrm{OH}, \mathrm{C}=\mathrm{O}$, and $\mathrm{C}-\mathrm{O}-\mathrm{C}$ ) around the surface. The chemical bonding states of the functional carbon and oxygen groups of the G-tags were also determined, as shown in Figs. 3d and S1, and Table S1. Figure 3d and Table S1 show the chemical composition, atomic ratio, and relative areas of the functional groups calculated by deconvoluting the C1s peaks. The G-tags showed C1s peak binding energies of $284.7^{34,35}$. The G-tags showed the $-\mathrm{C}-\mathrm{O}$ $(286.2 \mathrm{eV}), \mathrm{C}-\mathrm{O}-\mathrm{C}=\mathrm{O}(287.3 \mathrm{eV})$, and $\mathrm{O}=\mathrm{C}-\mathrm{O}-\mathrm{H}(288.9 \mathrm{eV})$ peaks in the $\mathrm{C} 1 \mathrm{~s}$ spectrum ${ }^{34}$. These functional groups improve the solubility and stability of the G-tags in an aqueous system. The presence of oxygen-containing functional groups on the surfaces of G-tags will increase the surface properties and the potential for application in various areas, including drug delivery, biosensing, and biomedical imaging. On the basis of the above results, a possible
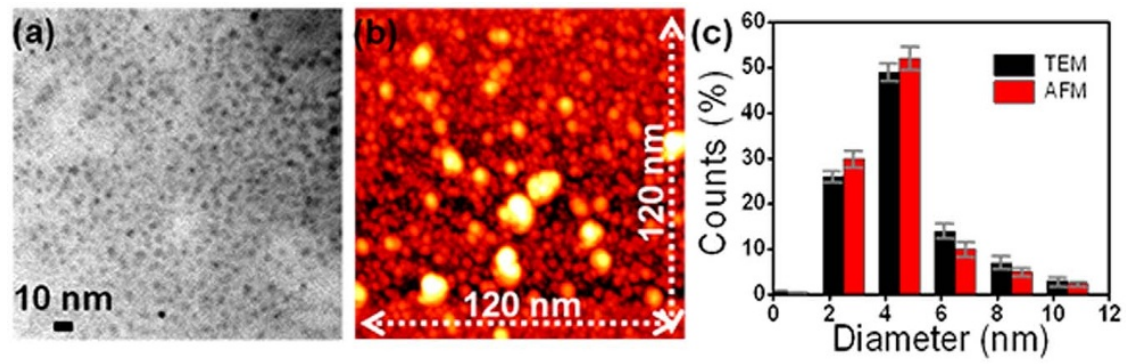

Figure $2 \mid$ The morphological features of the G-tags. (a) High resolution TEM, (b) AFM images, and (c) corresponding size distribution plot of G-tags. 

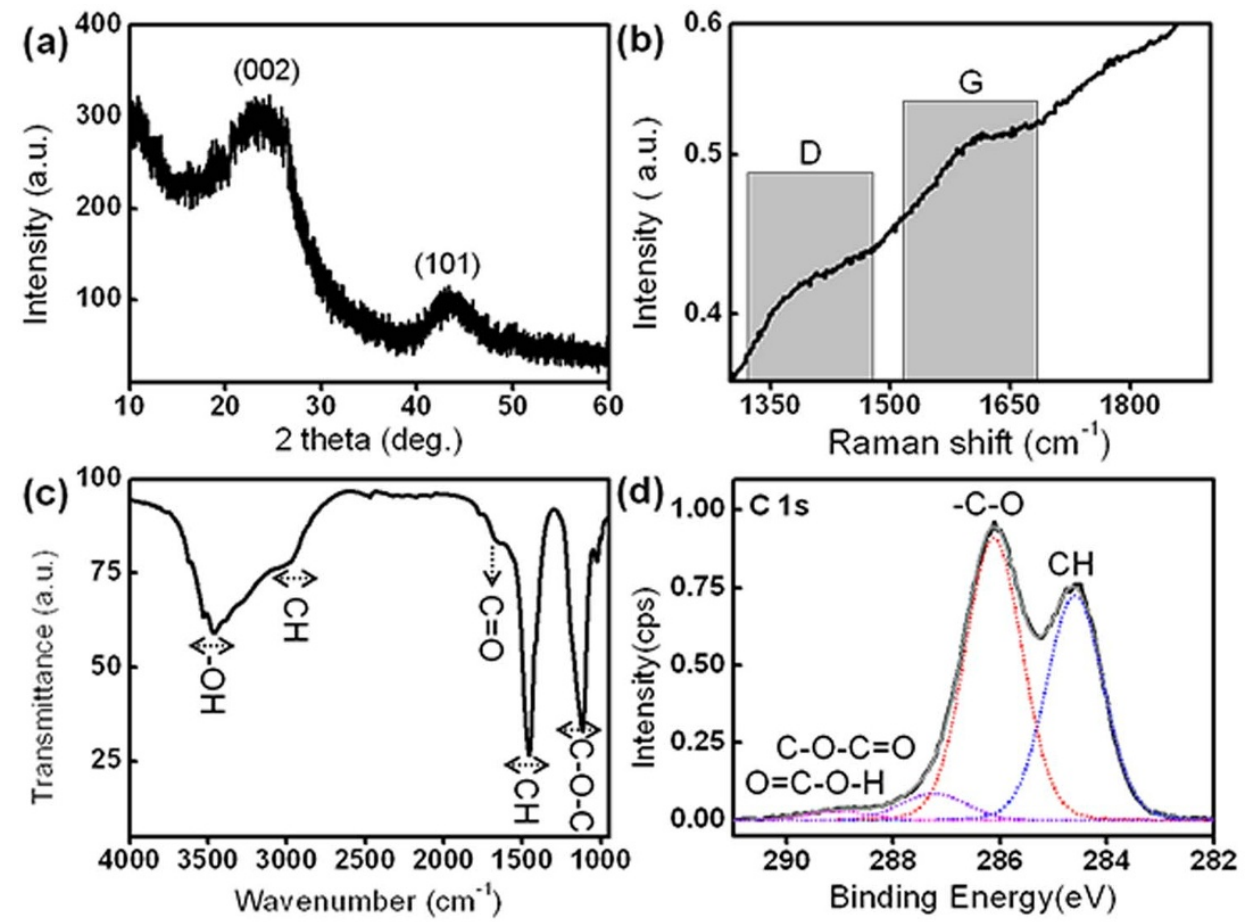

Figure 3 The structural properties of G-tags. (a) XRD pattern, (b) Raman spectrum, (c) FTIR spectrum and (d) XPS spectrum of the C1s region of Gtags.

mechanism for the formation of G-tags can be proposed by taking into account a series of chemical and physical interactions ${ }^{32,33,36}$. The G-tags were synthesised from harmful cyanobacteria through our simple process, which includes dehydration, polymerisation, carbonisation, and passivation. Initially, the harmful cyanobacteria are composed of combinations of many organic molecules due to hydrogen bonding. The dehydration, polymerisation, and carbonisation sequentially occur during the ultrasound irradiation, thereby resulting in a short single burst of nucleation. The resulting nuclei then move by the diffusion of solutes towards the carbon nanoparticle surfaces. The surfaces of these G-tags are highly oxygen functionalized and do not require any further modification, producing surfacepassivated carbonaceous quantum dots, similarly corresponding to carbon dots from biomass-derived carbohydrate sources ${ }^{36,37}$.

To analyse the optical properties of the synthesised G-tags, a detailed photoluminescence (PL) study was performed using different excitation wavelengths, as shown in Fig. 4a; the black-purple lines are the PL spectra for excitation at $350-610 \mathrm{~nm}$. The wide photoluminescence emission area $(300-500 \mathrm{~nm})$ of the G-tags confirmed that the size distribution $(2-12 \mathrm{~nm})$ was small due to similar quantum effects and emission traps on the surfaces, as supported by the TEM and AFM images (Fig. 2). The PL spectra also display the characteristic feature that the maximum emission moves to a longer wavelength as the excitation wavelength increases. Thus, the G-tags exhibited different emission colours, including blue to red, when they were excited by different wavelengths $(350-610 \mathrm{~nm})$ of light. We found that the intensity decreased when the $\mathrm{pH}$ value of the solution was higher or lower than 6-8. However, this change was nullified when the $\mathrm{pH}$ value was adjusted back to this optimal value (Fig. 4b). It was also observed that the photoluminescence intensity of the Gtags derived from harmful cyanobacteria was maximal at $\mathrm{pH} \mathrm{6-8,}$ with the intensity decreasing significantly (15-72\% decrease) with a slight blue shift upon changing to either an acidic or a basic solution. According to different $\mathrm{pH}$ microenvironment, the C-dots photostability for emitted fluorescence intensity could be variant ${ }^{38,39}$. However, the photoluminescence intensity in this study did not change significantly in the $\mathrm{pH}$ range $6-8$, indicating the potential bio-medical application of the present $\mathrm{C}$-dots in wide range of $\mathrm{pH}$. To evaluate the potential application of G-tags as fluorescent labels, the fluorescence stability of G-tags was investigated in aqueous conditions for $240 \mathrm{~h}$ (Fig. 4c). The G-tags had excellent photostability; their photoluminescence intensity decreased slowly $(\sim 8 \%)$ under
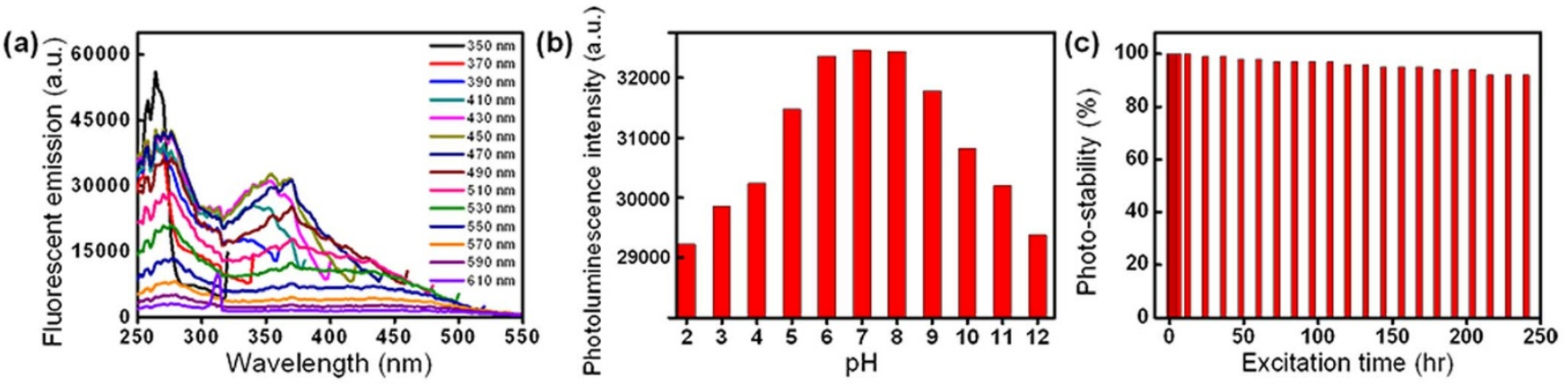

Figure $4 \mid$ The optical properties of G-tags. (a) PL emission spectra of G-tags in water, (b) fluorescence responses of G-tags towards various pH values and (c) fluorescence stability of G-tags. 

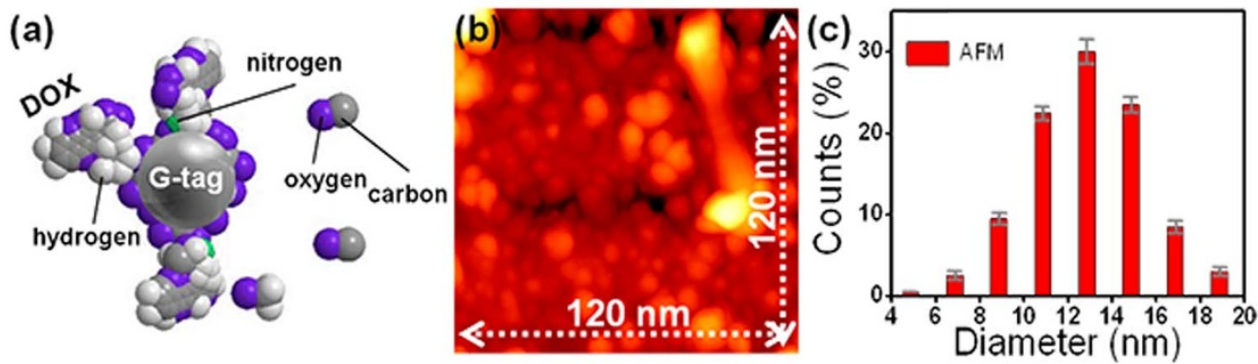

Figure $5 \mid$ Design and structural properties of T-tags. (a) Schematic representation of T-tags, (b) AFM images of T-tags and (c) size distribution plot of T-tags.

continuous excitation with a Xe lamp for $240 \mathrm{~h}$. The fluorescence of most of the G-tags is stable against photobleaching in solution conditions. These results show that the G-tags have great potential for use in protein (or nucleic acid)-labelling and bioimaging studies ${ }^{40,41}$.

Design and structural properties of T-tags. The design of the T-tags is shown schematically in Fig. 5a. We hypothesised that DOX could be loaded on the surfaces of G-tags through electrostatic interactions between positively charged DOX and the negatively charged G$\operatorname{tags}^{13,42}$. The AFM image showed that the T-tag nanoparticles had a uniform spherical shape with a diameter of 11-15 nm, ranging in most of the particles being $<19 \mathrm{~nm}$ (Figs. $5 \mathrm{~b}$ and $5 \mathrm{c}$ ). The average particle size $(14.9 \mathrm{~nm})$ of the T-tags was greater than that of G-tags $(5.7 \mathrm{~nm})$, as shown in Figs 2 and $5 \mathrm{~b}$. The FTIR spectra of $\mathrm{O}-\mathrm{H}$ at $3500 \mathrm{~cm}^{-1}$ and the stretching vibration bands of $\mathrm{C}-\mathrm{O}-\mathrm{C}$ at $1100 \mathrm{~cm}^{-1}$ showed the presence of oxygen-containing functional groups, indicating partial oxidation of the T-tags (Fig. S2) ${ }^{43}$. The peaks noted at approximately $1700 \mathrm{~cm}^{-1}$ indicated the existence of carbonyl $(\mathrm{C}=\mathrm{O})$ groups, which in turn indicates that the surfaces of the T-tags are partially oxidised. The T-tags also exhibited a new sharp peak associated with amide linkages (-CONH-) found at $1700 \mathrm{~cm}^{-1}$, indicating abundant interactions between DOX and adjacent $\mathrm{G}$-tags by the amide linkages ${ }^{43}$.

Cytotoxicity, pharmaceutical activity, and imaging of G- and Ttags in cells. For biomedical purposes, toxicity is a critical factor to be considered when evaluating a potential therapeutic agent. To evaluate the cytotoxicity of the G- and T-tags, the relative viabilities of normal [CHO-K1 (ovary; Chinese hamster) and COS-7 (kidney, African green monkey)] and cancer (HepG2 and MCF-7) cells exposed to G- and T-tags were measured using the MTT assay (Fig. 6). Figure 6a shows the cell viability after incubation with G-tags at a concentration of $0-4 \mathrm{mg} / \mathrm{mL}$ for $24 \mathrm{~h}$. After $24 \mathrm{~h}$ of incubation, the cell viability decreased with less than $16-18 \%$ when concentrations of $4 \mathrm{mg} / \mathrm{mL}$ of G-tags were used. These observations clearly indicate that, over a period of $24 \mathrm{~h}$, G-tags (at a concentration up to $2 \mathrm{mg} / \mathrm{mL}$ ) did not exert any cytotoxic effects on the cells. At lower concentrations there was a slight concentrationdependent reduction in cell viability. Importantly, at the concentrations typically used in bioimaging applications $(<2 \mathrm{mg} / \mathrm{mL})$, the cytotoxicity of the G-tags was found to be marginal. For pharmaceutical activity of T-tags, DOX as an anticancer drug was used to kill liver and breast cancer (Fig. 6b and Fig. S3) ${ }^{42,43}$, where DOX is wellknown as one of representative anticancer drugs and kills cancer cells selectively at a certain concentration ${ }^{44,45}$. The anticancer activity and imaging capability of T-tags was evaluated by incubating them with HepG2 and MCF-7 cells to confirm simultaneous pharmaceutical activities and imaging. The MTT cell proliferation assay demonstrates that the cytotoxicity of T-tags is greater in cancer cells (HepG2 and MCF-7) than normal cells (CHO-K1 and COS-7) when $1 \mathrm{mg} / \mathrm{mL}$ of T-tags was used (cellular viability: $26.4 \% \pm$ $1.52 \%$ for HepG2 and $30.4 \% \pm 1.96 \%$ for MCF-7 versus $89.3 \% \pm$
$2.17 \%$ for CHO-K1 and $93.1 \% \pm 3.82 \%$ for COS-7; mean \pm S.E., $\mathrm{N}=$ 5; probability value $p<0.005$ ) (Fig. 6b). The G-tags alone had no inherent cytotoxicity to HepG2 and MCF-7 cells (cellular viability: 98.3\% $\pm 1.93 \%$ for HepG2 and $97.2 \% \pm 2.01 \%$ for MCF-7; mean \pm S.E., $\mathrm{N}=5$; $p<0.005$; Fig. $6 \mathrm{a}$ ) when $1 \mathrm{mg} / \mathrm{mL}$ of T-tags was used.

A two-photon laser scanning confocal microscope operating at a $380 \mathrm{~nm}$ excitation wavelength was used to excite the T-tags (Fig. 7). HepG2 cells alone showed almost no background fluorescence upon excitation at $380 \mathrm{~nm}$ in a control experiment. After incubation for $24 \mathrm{~h}$ with HepG2, the T-tags fluorescence signal appeared mainly in the cytoplasm, while the free G-tags were observed to translocate into the nuclei due to their small size. As shown in Fig. 7c and d, the schematic diagram represents the principle of translocated fluorescence probes to indicate the probes' location in the different
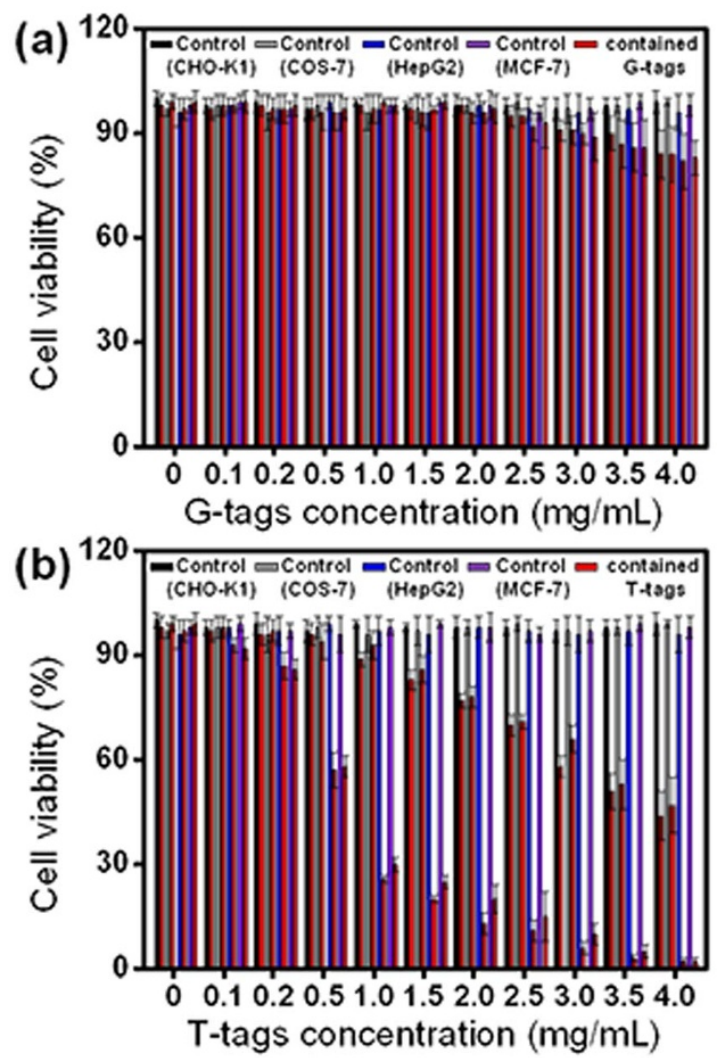

Figure 6 Cytotoxicity and pharmaceutical studies of G- and T-tags. To evaluate the cytotoxicity of the (a) G- and (b) T-tags, the relative viabilities of normal [CHO-K1 (ovary; Chinese hamster) and COS-7 (kidney, African green monkey)] and cancer [HepG2 (hepatocellular liver carcinoma, human) and MCF-7 (breast cancer, human)] cells exposed to G- and T-tags were measured using the MTT assay. The data is presented as mean \pm S.E. of five replicates. 

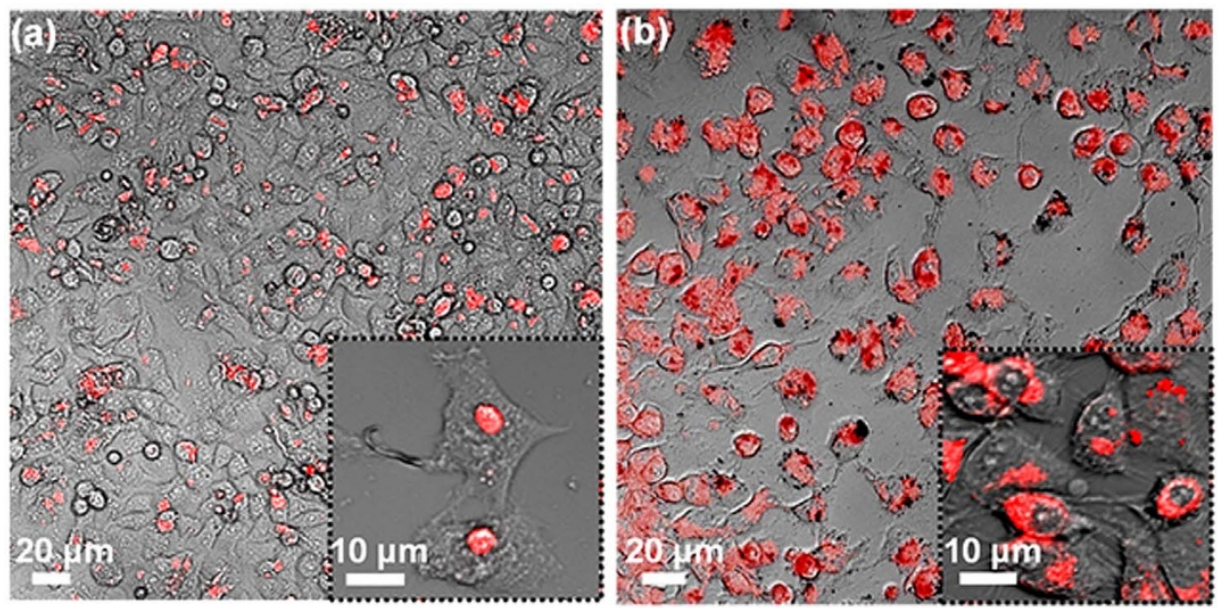

(c)

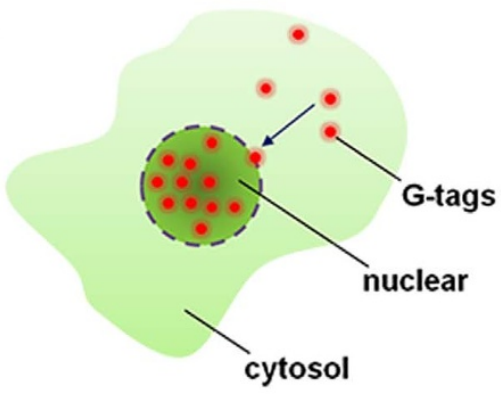

(d)

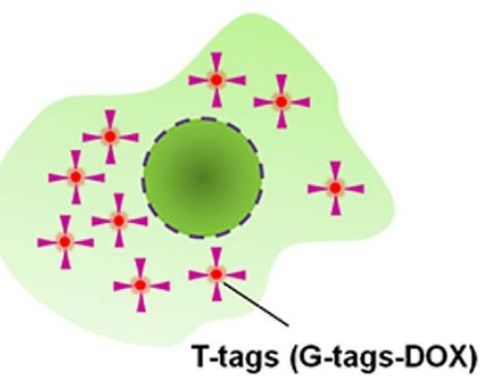

Figure 7 Imaging of G- and T-tags. Fluorescence images of HepG2 cells incubated for $24 \mathrm{~h}$ with the (a) G- and (b) T-tags, and schematic illustration of topical location for (c) G- and (d) T-tags in nucleus and cytosol of cancer cells, respectively.

regions of the cell. Because T-tags are composed of highly biocompatible carbon particles, no significant toxicity was observed in normal and cancer cells when concentrations of $<0.5 \mathrm{mg} / \mathrm{mL}$ of T-tags were used. T-tags showed remarkable inhibition of cancer growth of approximately $67.9 \%$ relative to the control when a concentration of $1 \mathrm{mg} / \mathrm{mL}$ was used (Fig. 6). For practically feasible applications, in-vivo mice assay of T-tags with $0.01 \mathrm{mg} / \mathrm{mL}$ resulted in enhanced anticancer effect, compared to free DOX for 12 days after syringe injection (Fig. 8). The inhibition effect (\%) of T-tags and free DOX treatment versus no treatment showed about $\sim 68.66$ and $\sim 53.12 \%$ after 24 days, resulting in enhancement of $15.54 \%$ discrepancy.

\section{Discussion}

The generation and disposal of harmful cyanobacteria is a global issue that impacts humans and the ecosystem. Therefore, finding ways to recycle harmful cyanobacteria into useful products is needed for environmental sustainability and economical benefit. In terms of synthesis, G-tags can be fabricated uniformly from harmful cyanobacteria biomass using simple procedures. The highly oxygen-functionalized surface of $\mathrm{G}$-tags has a number of functional groups $(-\mathrm{OH}$, $\mathrm{C}=\mathrm{O}$ and $\mathrm{O}=\mathrm{C}-\mathrm{O}-\mathrm{H})$ that enable the attachment of anticancer drugs without any further modification, resulting in water-soluble properties and cancer cell uptake of G-tags. The G-tags have excellent photostability under long-term aqueous conditions for $240 \mathrm{~h}$ and low cytotoxicity $(<2 \mathrm{mg} / \mathrm{mL})$. Moreover, doxorubicin-conjugated G-tags (T-tags at $0.1 \mathrm{mg} / \mathrm{mL}$ ) induced death in cancer cells (HepG2 [hepatocellular liver carcinoma, human] and MCF-7 [breast cancer, human]) at a higher rate than unlabelled with G-tags, indicating that the loaded DOX retains its pharmaceutical activity. These data suggest that DOX is released from the T-tags after endocytic uptake, possibly because the dissociation constant favors the release of DOX in an intracellular environment ${ }^{46-48}$. Alternatively, the delivery of DOX from the T-tags may occur through gradual degradation

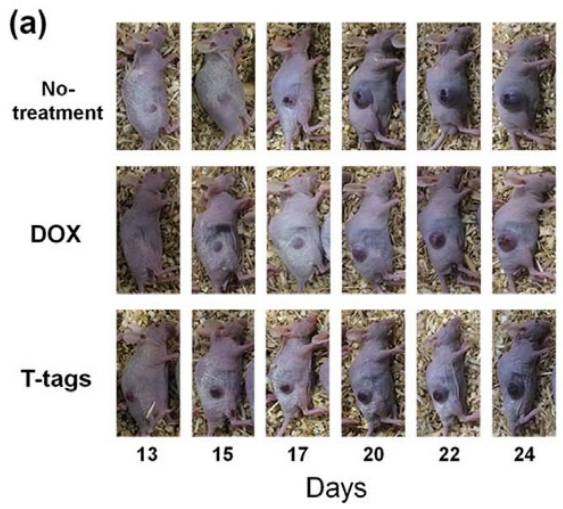

(b)

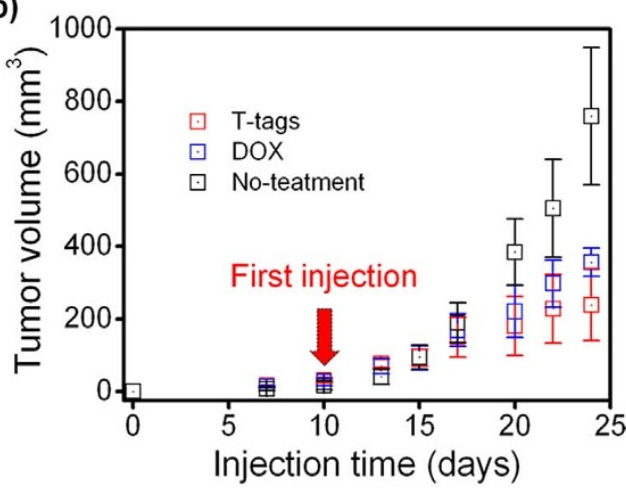

Figure $8 \mid$ Anticancer effect of T-tags on nude mice for 24 days. (a) A dica-image and (b) volume inhibition $\left(\mathrm{mm}^{3}\right)$ of cancer cell of no-treatment, free DOX, and T-tags treatments. 
of the G-tags by endonucleases in the lysosome following cellular uptake $^{46,47}$. Based on the results of in-vivo test, inhibition cancer cell growth of T-tags was improved compared to free DOX injection. However, the enhanced mechanism of T-tags is currently investigated with supported bioimaging. As a result, the ability to engineer novel methods to produce large-scale fluorescent G- or Ttags from an abundant natural resource such as harmful algal bloom could have significant implications in stimulating domestic energy economies, environmental problems, and human disease.

\section{Methods}

Reagents and materials. All reagents were purchased from Sigma-Aldrich (Milwaukee, WI, USA). Doxorubicin hydrochloride (DOX), fetal bovine serum (FBS), Dulbecco's modified Eagle's medium (DMEM), RPMI-1640, antibioticantimycotic, L-glutamine, and nonessential amino acids were obtained from Biowest (Lewes, UK). Ultrapure water $(18.2 \mathrm{M} \Omega / \mathrm{cm})$ from a Milli-Q ultrapure system was used in this study. The $\mathrm{pH}$ values of phosphate buffer solutions were in the range of 3.0-10.0. The phosphate-buffered saline (PBS; $1 \times, 1 \mathrm{~L}, \mathrm{pH} 7.4)$ contained $\mathrm{NaCl}(8 \mathrm{~g})$, $\mathrm{KCl}(0.2 \mathrm{~g}), \mathrm{Na}_{2} \mathrm{HPO}_{4}(1.44 \mathrm{~g})$, and $\mathrm{KH}_{2} \mathrm{PO}_{4}(0.24 \mathrm{~g})$

Synthesis of G-tags and preparation of DOX loaded G-tags (T-tags). To fabricate the G-tags with our simple method, mainly cyanobacteria species were collected from Nakdong river, located in the southeast part of Korea. The harmful cyanobacteria/ ethanol (100 kilograms of cyanobacteria per 20 litres of $10 \%$ ethanol) solution was treated for $90 \mathrm{~min}$ with ultrasound at $40 \mathrm{kHz}$ (Ultrasonics UC-05, Lab Companion, Korea). The solution, after separating $50-\mathrm{mL}$ Falcon ${ }^{\circledR}$ tubes, was then centrifuged at $4500 \mathrm{rpm}$ for $5 \mathrm{~min}$ to remove large or agglomerated particles using the centrifuge (GYROZEN, 1236MG, Seoul, Korea). The supernatant (containing G-tags) was filtered twice through a $0.22-\mu \mathrm{m}$ membrane to remove large particles and further treated with dialysis (MWCO 8,000 Da, Sigma-Aldrich) in ultrapure water for 6 hours by UV-Vis spectrophotometer (UV-1800, Shimadzu, Japan) at $484 \mathrm{~nm}$ wavelength, resulting in $95 \%$ of the DOX loading efficiency. Finally, it was dried at $45^{\circ} \mathrm{C} .2 \mathrm{~g}$ of G-tags was mixed with $0.25 \mathrm{~g}$ of DOX in ultrapure water $(1 \mathrm{~g} / \mathrm{L})$. After stirring for $24 \mathrm{~h}$ under dark conditions at $4{ }^{\circ} \mathrm{C}$, the T-tags were centrifuged and washed with ultrapure water, followed by freeze-drying.

Sample characterisation. The morphological features and size of the G-tags were analysed by AFM (VEECO Instrument, USA) and HR-TEM (JEM 2200, Japan). For the AFM analysis, $100 \mu \mathrm{L}$ of the G-tag solution was placed on a silicon wafer. The silicon wafer was air-dried overnight, and the remaining solution was dispersed using an air gun. The HR-TEM specimens were prepared by drop-casting $10 \mu \mathrm{L}$ of the Gtag solution on a 300-mesh carbon-coated copper TEM grid with a carbon film, followed by drying at room temperature. PL spectroscopy with a changeable UV transilluminator (DUT-260; Core Bio System, Korea) at excitations (350, 370, 390 $410,430,450,470,490,510,530,550,570,590$, and $610 \mathrm{~nm})$ and emission (250$550 \mathrm{~nm}$ ) was used to measure the optical properties of G-tags. A Raman microscopy system (NT-MDT NTEGRA system, USA) was used to analyse the air-dried G-tags coated on a silica wafer. FT-IR spectra were investigated on a JASCO FTIR 470. Each spectrum was recorded from 4000 to $700 \mathrm{~cm}^{-1}$ using 12 scans at a resolution of $4 \mathrm{~cm}^{-1}$. The G-tag crystalline structure was measured by XRD (Rigaku RDA-cA Xray diffractometer, Japan) using $\mathrm{Cu} \mathrm{K} \alpha$ radiation and a nickel filter. High resolution$\mathrm{X}$-ray photoelectron spectroscopy (HR-XPS) with monochromatic Al K $\alpha$ X-ray radiation $(\mathrm{hv}=1486.6 \mathrm{eV}$ ) operated at $120 \mathrm{~W}$ (Kratos Analytical, AXIS Nova, Manchester, UK) was used to investigate the surface properties of the samples. The shift of the binding energy as a result of relative surface charging was corrected using the $\mathrm{C} 1 \mathrm{~s}$ level at $284.6 \mathrm{eV}$ as an internal standard.

Cytotoxicity and pharmaceutical testing of the samples. The cytotoxicities of the samples were evaluated by the MTT assay. Briefly, CHO-K1, COS-7, HepG2, and MCF-7 cells were seeded in a 96-well plate at a density of $8 \times 10^{3}$ cells per well and cultured in a humidified incubator at $37^{\circ} \mathrm{C}$ for $24 \mathrm{~h}$ under a $5 \% \mathrm{CO}_{2}$ atmosphere in DMEM and/or RPMI-1640 supplemented with 10\% FBS and $1 \%$ penicillinstreptomicin antibiotics. Fresh medium containing an increasing concentration of DOX, G- and T-tags was added to each well and incubated for $24 \mathrm{~h}$. Then, $20 \mu \mathrm{L}$ of a $0.2 \mathrm{mg} / \mathrm{mL}$ MTS solution in DMEM and/or RPMI-1640 was added to each well and incubated at $37^{\circ} \mathrm{C}$ for $2 \mathrm{~h}$. Finally, the optical density was measured at $490 \mathrm{~nm}$ with an absorbance microplate reader (EMax microplate reader, Bucher Biotec AG, Basel, Switzerland).

Imaging of G- and T-tags. HepG2 cells were seeded in DMEM containing high glucose supplemented with $10 \%$ FBS, 100 units $/ \mathrm{mL}$ penicillin, and $100 \mu \mathrm{g} / \mathrm{mL}$ streptomycin. When the HepG 2 cells were grown at stationary phase, the cells $(\sim 1 \times$ $10^{7} \mathrm{cell} / \mathrm{mL}$ ) were dispersed within replicate 6-well plates to a total volume of $100 \mu \mathrm{L} /$ well and maintained at $37^{\circ} \mathrm{C}$ in a $5 \% \mathrm{CO}_{2}$ incubator for $24 \mathrm{~h}$. The culture medium was then removed, and the cells were incubated in culture medium containing free G- and $\mathrm{T}$-tags for $24 \mathrm{~h}$, followed by washing with the culture medium. Fluorescence images were obtained using a confocal microscope (LSM 510META; Carl Zeiss).
In-vivo anticancer assay of T-tags in mice. All experiments in mice were in accordance with relevant institutional guidelines and regulations, and were submitted to a protocol approval by the Animal Ethics Committee, the Korea Basic Science Institute (KBSI). Male nude mice weighing 20-22 g were purchased from Daehan Biolink Co. Ltd (Korea). The mice were allowed at least 1 week to adapt to their environment before the experiments. Eight-week-old male nude mice were injected s.c. on the right rear flank area with $1.5 \times 10^{6} \mathrm{CT} 26$ cancer cells in $100 \mu \mathrm{L}$ of serum free DMEM medium. When the xenografts reached about $25-30 \mathrm{~mm}^{3}$, tumorbearing mice were divided into three groups, six in each group, and treated as follows: no treatment; injection of DOX $(0.01 \mathrm{mg} / \mathrm{mL})$; and injection of T-tags $(0.01 \mathrm{mg} / \mathrm{mL})$. All injections were with $50 \mu \mathrm{L}$ volume of PBS and performed via tail vain, one dose per every three days, totally in four doses. Tumor measurements were performed by using an electronic vernier caliper every two or three days, and tumor volumes were calculated by using the following equation: $\mathrm{V}\left(\mathrm{mm}^{3}\right)=\mathrm{ab}^{2} / 2$, where $\mathrm{a}$ is the largest diameter and $\mathrm{b}$ is the diameter perpendicular to $\mathrm{a}$

1. Ashley, C. E. et al. The targeted delivery of multicomponent cargos to cancer cells by nanoporous particle-supported lipid bilayers. Nat. Mater. 10, 389-397 (2011).

2. Gao, X. et al. In vivo cancer targeting and imaging with semiconductor quantum dots. Nat. Biotechnol. 22, 969-976 (2004).

3. Guduru, R. et al. Magneto-electric nanoparticles to enable field-controlled highspecificity drug delivery to eradicate ovarian cancer cells. Sci. Rep. 3, 2953 (1-8) (2013).

4. Guo, C.X. et al. A new class of fluorescent-dots: long luminescent lifetime bio-dots self-assembled from DNA at low temperatures. Sci. Rep. 3, 2957 (1-6) (2013).

5. Kim, J. et al. Targeted delivery of nanoparticles to ischemic muscle for imaging and therapeutic angiogenesis. Nano Lett. 11, 694-700 (2011).

6. Song, J., Zhou, J. \& Duan, H. Self-assembled plasmonic vesicles of SERS-encoded amphiphilic gold nanoparticles for cancer cell targeting and traceable intracellular drug delivery. J. Am. Chem. Soc. 134, 13458-13469 (2012).

7. Hong, H. et al. Cancer-targeted optical imaging with fluorescent zinc oxide nanowires. Nano Lett. 11, 3744-3750 (2011).

8. Li, X. et al. Amphiphilic multiarm star block copolymer-based multifunctional unimolecular micelles for cancer targeted drug delivery and MR imaging. Biomater. 32, 6595-6605 (2011).

9. Du, J. Z., Du, X. J., Mao, C. Q. \& Wang, J. Tailor-made dual pH-sensitive polymer doxorubicin nanoparticles for efficient anticancer drug delivery. J. Am. Chem. Soc. 133, 17560-17563 (2011).

10. Park, K. et al. New generation of multifunctional nanoparticles for cancer imaging and therapy. Adv. Funct. Mater. 19, 1553-1566 (2009).

11. Brown, S. D. et al. Gold nanoparticles for the improved anticancer drug delivery of the active component of oxaliplatin. J. Am. Chem. Soc. 132, 4678-4684 (2010).

12. Bagalkot, V. et al. Quantum dot-aptamer conjugates for synchronous cancer imaging, therapy, and sensing of drug delivery based on bi-fluorescence resonance energy transfer. Nano Lett. 7, 3065-3070 (2007).

13. Yu, M. K. et al. Drug-loaded superparamagnetic iron oxide nanoparticles for combined cancer imaging and therapy in vivo. Angew. Chem. Int. Edit. 47, 5362-5365 (2008).

14. Yang, X. et al. cRGD-functionalized, DOX-conjugated, and ${ }^{64} \mathrm{Cu}$-labeled superparamagnetic iron oxide nanoparticles for targeted anticancer drug delivery and PET/MR imaging. Biomater. 32, 4151-4160 (2011).

15. Guo, Y. et al. in vivo imaging and drug storage by quantum-dot-conjugated carbon nanotubes. Adv. Funct. Mater. 18, 2489-2497 (2008).

16. Xiao, Y. et al. Multifunctional unimolecular micelles for cancer-targeted drug delivery and positron emission tomography imaging. Biomater. 33, 3071-3082 (2012).

17. Cheng, Z. et al. Multifunctional nanoparticles: cost versus benefit of adding targeting and imaging capabilities. Science 338, 903-910 (2012).

18. Loo, C. Lowery, A., Halas, N., West, J. \& Drezek, R. Immunotargeted nanoshells for integrated cancer imaging and therapy. Nano Lett. 5, 709-711 (2005).

19. Chen, Y.et al. Core/shell structured hollow mesoporous nanocapsules: a potential platform for simultaneous cell imaging and anticancer drug delivery. ACS Nano 4, 6001-6013 (2010).

20. Liong, M. et al. Multifunctional inorganic nanoparticles for imaging, targeting, and drug delivery. ACS Nano 2, 889-896 (2008).

21. Sahu, S., Behera, B., Maiti, T. K. \& Mohapatr, S. Simple one-step synthesis of highly luminescent carbon dots from orange juice: application as excellent bioimaging agents, Chem. Commun. 48, 8835-8837 (2012).

22. Hsu, P. C., Shih, Z. Y., Lee, C. H. \& Chang, H. T. Synthesis and analytical applications of photoluminescent carbon nanodots, Green Chem. 14, 917-920 (2012).

23. Park, S. Y. et al. Photoluminescent green carbon nanodots from food-wastederived sources: Large-scale synthesis, properties, and biomedical applications. ACS Appl. Mater. Interfaces 6, 3365-3370 (2014).

24. Park, J. H. et al. Biodegradable luminescent porous silicon nanoparticles for in vivo applications, Nat. Mater. 8, 331-336 (2009)

25. Ray, S. C., Saha, A., Jana, N. R. \& Sarkar, R. Fluorescent carbon nanoparticles: synthesis, characterization, and bioimaging application, J. Phys. Chem. C 113, 18546-18551 (2009).

26. Yang, S. T. et al. Carbon dots for optical imaging in vivo, J. Am. Chem. Soc. 131, 11308-11309 (2009). 
27. Sun, Y. P. et al. Quantum-sized carbon dots for bright and colorful photoluminescence, J. Am. Chem. Soc. 128, 7756-7757 (2006).

28. Kim, J., Park, J., Kim, H., Singha, K. \& Kim, W. J. Transfection and intracellular trafficking properties of carbon dot-gold nanoparticle molecular assembly conjugated with PEI-pDNA, Biomater. 34, 7168-7180 (2013).

29. Zhou, L. et al. Carbon nanodots as fluorescence probes for rapid, sensitive, and label-free detection of $\mathrm{Hg}^{2+}$ and biothiols in complex matrices, Chem. Commun. 48, 1147-1149 (2012).

30. Welsher, K. et al. A route to brightly fluorescent carbon nanotubes for nearinfrared imaging in mice, Nat. Nanotechnol. 4, 773-780 (2009).

31. Qu, S. et al. A biocompatible fluorescent ink based on water-soluble luminescent carbon nanodots. Angew. Chem. Int. Edit. 124, 12381-12384 (2012).

32. Li, H., Kang, Z., Liu, Y. \& Lee, S. T. Carbon nanodots: synthesis, properties and applications. J. Mater. Chem. 22, 24230-24253 (2012).

33. Jeong, J. et al. Color-tunable photoluminescent fullerene nanoparticles. Adv. Mater. 24, 1999-2003 (2012).

34. Lee, H. U. et al. Long-term aging characteristics of atmospheric-plasma-treated poly(E-caprolactone) films and fibres. Polym. Degrad. Stab. 96, 1204-1209 (2011).

35. Gonzalez, II. E. \& Hicks, R. F. Surface analysis of polymers treated by remote atmospheric pressure plasma. Langmuir 26, 3710-3719 (2010).

36. Baker, S. N. \& Baker, G. A. Luminescent carbon nanodots: emergent nanolights. Angew. Chem. Int. Edit. 49, 6726-6744 (2010).

37. Sk, M. P. et al. Presence of amorphous carbon nanoparticles in food caramels. Sci. Rep. 2, 383 (1-5) (2012).

38. Jaiswal, A., Ghosh, S. S. \& Chattopadhyay, A. One step synthesis of C-dots by microwave mediated caramelization of poly(ethylene glyccol). Chem. Commun. 48, 407-409 (2012).

39. Tan, M. et al. Enhanced photoluminescence and characterization of multicolor carbon dots using plant soot as a carbon source. Talanta 115, 950-956 (2013).

40. Lu, J. et al. One-pot synthesis of fluorescent carbon nanoribbons, nanoparticles, and graphene by the exfoliation of graphite in ionic liquids. ACS Nano 3, 2367-2375 (2009).

41. Yang, Z. C. et al. Intrinsically fluorescent carbon dots with tunable emission derived from hydrothermal treatment of glucose in the presence of monopotassium phosphate. Chem. Commun. 47, 11615-11617 (2011)

42. Liu, Z., Sun, X., Nakayama-Ratchford, N. \& Dai, H. Supramolecular chemistry on water-soluble carbon nanotubes for drug loading and delivery. ACS Nano 1, 50-56 (2007).

43. Zhang, Q. et al. Tailoring the interlayer interaction between doxorubicin-loaded graphene oxide nanosheets by controlling the drug content. Carbon 51, 164-172 (2013).

44. Lee, I.-H. et al. A duplex oligodeoxynucleotide-dendrimer bioconjugates as a novel delivery vehicle for doxorubicin in in vivo cancer therapy. J. Control. Release 155, 88-95.
45. Chen, Y. et al. In vivo distribution and antitumor activity of doxorubicin-loaded $\mathrm{N}$-isopropylacrylamide-co-methacrylic acid coated mesoporous silica nanoparticles and safety evaluation. Eur. J. Pharm. Biopharm. 85, 406-412.

46. Bagalkot, V., Farokhzad, O. C., Langer, R. \& Jon, S. An aptamer-doxorubicin physical conjugate as a novel targeted drug-delivery platform. Angew. Chem. Int. Edit. 45, 8149-8152 (2006).

47. Yang, X. et al. Multifunctional stable and $\mathrm{pH}$ responsive polymer vesicles formed by heterofunctional triblock copolymer for targeted anticancer drug delivery and ultrasensitive MR imaging. ACS Nano 4, 6805-6817 (2007).

48. Di, W. et al. Single-phased luminescent mesoporous nanoparticles for simultaneous cell imaging and anticancer drug delivery. Biomater. 32, 7226-7233 (2011)

\section{Acknowledgments}

This research was supported by the Converging Research Center Program through the Ministry of Science, ICT and Future Planning, Korea (2013K000163).

\section{Author contributions}

H.U.L., S.Y.P., E.S.P. and J.L. designed the project and organized the entire research. H.U.L., S.Y.P., E.S.P., Y.C.L. and J.L. and wrote the manuscript. H.U.L. and E.S.P. carried out the sample preparation and characterization. B.S. performed the XPS analysis. S.C.L., J.L. Y.S.H. and Y.K.O. performed the AFM, TEM, Raman and PL analysis. Y.C.L., K.S.K., M.I.L., H.G.P. and S.H.C performed the cytotoxicity, pharmaceutical testing and cell imaging. S.Y.L. and K.B.L. conducted in-vivo mice experiment. All authors discussed the results and commented on the manuscript.

\section{Additional information}

Supplementary information accompanies this paper at http://www.nature.com/ scientificreports

Competing financial interests: The authors declare no competing financial interests.

How to cite this article: Lee, H.U. et al. Photoluminescent carbon nanotags from harmful cyanobacteria for drug delivery and imaging in cancer cells. Sci. Rep. 4, 4665; DOI:10.1038/ srep04665 (2014).

(c) (1) (2) This work is licensed under a Creative Commons Attribution-NonCommercialShareAlike 3.0 Unported License. The images in this article are included in the article's Creative Commons license, unless indicated otherwise in the image credit; if the image is not included under the Creative Commons license, users will need to obtain permission from the license holder in order to reproduce the image. To view a copy of this license, visit http://creativecommons.org/licenses/by-nc-sa/3.0/ 